\title{
Evaluation of EGM08 using GPS and leveling heights in Greece
}

\author{
C. Kotsakis, K. Katsambalos, D. Ampatzidis \\ Department of Geodesy and Surveying \\ School of Engineering, Aristotle University of Thessaloniki \\ Univ. Box 440, Thessaloniki 541 24, Greece
}

\author{
M. Gianniou \\ Ktimatologio S.A. (Hellenic Cadastre) \\ 339 Mesogion Ave.
}

Athens 152 31, Greece

\begin{abstract}
This paper presents an overview of the evaluation results for the new Earth Gravitational Model (EGM08) that was recently released by the US National Geospatial-Intelligence Agency, using GPS and leveled orthometric heights in the area of Greece. Various comparisons of geoid undulations obtained from the EGM08 model, other combined geopotential models and GPS/leveling data have been performed in both absolute (at individual points) and relative (for baselines of varying length) sense. The test network covers the entire part of the Greek mainland and it consists of more than 1500 benchmarks that belong to the Hellenic national triangulation network, with direct leveling ties to the Hellenic vertical reference frame. The spatial positions of these benchmarks have been recently determined at cm-level accuracy (with respect to ITRF2000) through an extensive national GPS campaign that was organized in the frame of the HEPOS project. Our results suggest that EGM08 offers a major improvement (more than 50\%) in the agreement level among geoidal, ellipsoidal and orthometric heights over the mainland part of Greece, compared to the performance of previous global geopotential models for the same area.
\end{abstract}

Keywords. EGM08, geoid, ellipsoidal heights, orthometric heights, GPS leveling.

\section{Introduction}

The recent release of the new Earth Gravitational Model EGM08 by the US National GeospatialIntelligence Agency (Pavlis et al. 2008) is undoubtedly a major breakthrough in global gravity field mapping. For the first time, a spherical harmonic (SH) model complete to degree and order 2159, with additional SH coefficients extending up to degree 2190 and order 2159 , is available for the Earth's external gravitational potential, leading to an unprecedented level of spatial sampling resolution $(\sim 9 \mathrm{~km})$ for the recovery of gravity field functionals over the entire globe. Such a revolutionary step contributes in a most successful way to the continuing efforts of the geodetic community dur- ing the last years (and after the launch of the satellite missions CHAMP and GRACE) for a highresolution and high-accuracy reference model of Earth's static (mean) gravity field. Furthermore, it provides an indispensable tool to support new gravity field studies and other Earth monitoring projects, especially in view of the upcoming GOCE mission (scheduled for launch in February 2009) and the ongoing development of the Global Geodetic Observing System (GGOS).

Following the official release of the EGM08 model, there is an expected strong interest among geodesists to quantify its actual accuracy with several validation techniques and 'external' data sets, independently of the estimation and error calibration procedures that were used for its development. In response to the above interest and as part of the related activities that have been coordinated by the IAG/IGFS Joint Working Group on the Evaluation of Global Earth Gravity Models, the objective of this paper is to present the results of the EGM08 validation tests that were performed in the area of Greece using GPS and leveled orthometric heights.

Our test network consists of 1542 control points that belong to the Hellenic national triangulation network, and which are directly tied to the Hellenic national vertical reference frame through spirit (and in some cases trigonometric) leveling surveys. These control points were recently re-surveyed during a national GPS campaign in the frame of the HEPOS project (more details to be given in Sect. 2) and their spatial positions have been estimated anew at $\mathrm{cm}$-level accuracy with respect to the ITRF2000 frame.

Key aspects of our study are the extensive national coverage and high spatial density of the underlying test network (see Fig. 1) which enabled us to identify the significant improvement that EGM08 yields, over other existing geopotential models, for the representation of gravity field features in certain mountainous areas around Greece (see Sect. 3). This is actually the first time that a detailed quality 
analysis for geopotential models is performed over the entire Hellenic mainland with the aid of precise GPS positioning. Hence, our study also provides a preliminary, yet reliable, assessment about the feasibility of EGM08 for determining orthometric height differences via GPS/geoid-based leveling techniques throughout Greece (see Sect. 4).

\section{Data sets}

All our evaluation tests and related results that are presented in the next sections refer to the network of 1542 GPS/leveling benchmarks shown in Fig. 1, which covers the entire mainland region of Greece with a relatively uniform spatial distribution. Note that control points with identified or suspected blunders (mainly in their orthometric heights that are provided by the Hellenic Military Geographic Service) have been removed from the following analysis and they are not included in this network.



Figure 1. Geographical distribution of the 1542 GPS/leveling benchmarks over the Greek mainland.

Although a large number of additional GPS/leveling benchmarks were also available over the Greek islands, they have been deliberately excluded from our current tests to avoid misleading systematic effects in the evaluation results due to unknown vertical datum differences between the various islands and the mainland region.

\section{Ellipsoidal heights (HEPOS Project)}

Due to current ongoing efforts for the enhancement of the spatial data infrastructure in Greece, a national GPS campaign was performed in 2007 to obtain a sufficient number of control points with accurately known 3D spatial positions in an ITRFtype coordinate system. These activities have been initiated by the Ministry for the Environment, Planning and Public Works and the financial support of the EU and the Hellenic State, and they are part of the HEPOS (Hellenic Positioning System) project that will lead to the launch of a modern satellitebased positioning service for cadastral, mapping, geodetic and other types of surveying applications in Greece. The entire project is coordinated by Ktimatologio S.A, a state-owned private sector firm that is responsible for the management of the Hellenic Cadastral system (see Gianniou 2008).

The aforementioned GPS campaign involved more than 2450 control stations within the existing national triangulation network (part of which are the 1542 points shown in Fig. 1) that will provide the basis for determining a precise datum transformation model between the official Hellenic Geodetic Reference Frame of 1987 (HGRF87) and other ITRF/ETRF-type frames. The actual fieldwork was performed within a 6-month period (March to September 2007) using twelve dual-frequency Trimble $5700 / 5800$ GPS receivers with Zephyr or R8 internal antennas. Thirty three of these points were used as base reference stations (with 24-hour continuous GPS observations) to obtain baseline solutions with the rest of the network points (observation time for each rover station ranged between 1-3 hours). In all cases, a 15 -sec sampling rate and an $15^{\circ}$ elevation cut-off angle were used for the data collection. After the processing of the GPS observations using EUREF/EPN ties and IGS precise orbits, the geocentric Cartesian coordinates of all above stations were determined in ITRF2000 (epoch: 2007.236) and their geometric heights were subsequently derived with respect to the GRS80 reference ellipsoid. The accuracy of the ellipsoidal heights ranges between $2-5 \mathrm{~cm}$, while the horizontal positioning accuracy is marginally better by $1-2 \mathrm{~cm}$ ( $1 \sigma$ level).

\section{Orthometric heights}

The orthometric heights for all points in our test network have been obtained through leveling survey ties to surrounding benchmarks of the national vertical reference frame. These local ties were performed in previous years from the Hellenic Military Geographic Service through spirit and trigonometric leveling techniques. Note that a large number of the 1542 control points is located in highly mountainous areas $(24 \%$ of the GPS/leveling benchmarks have orthometric heights $H>800 \mathrm{~m}$ ). The quality of the available orthometric heights in our test network is affected by two main factors: the internal accuracy and consistency of the Hellenic vertical datum (HVD), and the observation accuracy of the local leveling ties to the surrounding HVD benchmarks. 
Due to the absence of sufficient documentation, the actual accuracy of the orthometric heights at these points is largely unknown. Their values refer, in principle, to the Earth's equipotential surface that coincides with the mean sea level at the HVD's fundamental tide-gauge reference station located in Piraeus port (unknown $W_{o}$ value).

\section{GPS-based geoid undulations}

Based on the known ellipsoidal and orthometric heights, geoid undulations have been computed at the $1542 \mathrm{GPS} /$ leveling benchmarks of the test network according to the equation

$$
N^{G P S}=h-H
$$

The above values provide the 'external' dataset upon which the EGM08 validation tests will be performed. Note that low-pass filtering has not been applied to the pointwise $N^{G P S}$ heights. As a result, the effect of the omission error associated with all tested global geopotential models (GGMs) will be reflected in our evaluation results.

\section{GGM-based geoid undulations}

Geoid undulations have also been computed at the 1542 GPS/leveling benchmarks using several different GGMs. For the evaluation results presented herein we shall consider only the most recent 'mixed' GGMs which incorporate the contribution from various types of satellite data (CHAMP, GRACE, SLR), terrestrial gravity data, and satellite altimetry data; see Table 1.

Table 1. GGMs used for the evaluation tests at the 1542 GPS/leveling benchmarks.

\begin{tabular}{l|c|c}
\hline Models & $n_{\max }$ & Reference \\
\hline EGM08 & 2190 & Pavlis et al. (2008) \\
EIGEN-GL04C & 360 & Förste et al. (2006) \\
EIGEN-CG03C & 360 & Förste et al. (2005) \\
EIGEN-CG01C & 360 & Reigber et al. (2006) \\
GGM02C & 200 & Tapley et al. (2005) \\
EGM96 & 360 & Lemoine et al. (1998) \\
\hline
\end{tabular}

The determination of GGM geoid undulations was carried out through the general formula (Rapp 1997)

$$
N=\zeta+\frac{\Delta g^{F A}-0.1119 H}{\bar{\gamma}} H+N_{o}
$$

where $\zeta$ and $\Delta g^{F A}$ denote the height anomaly and free-air gravity anomaly, respectively, that are computed from the corresponding series expansions (up to $n_{\max }$ ) based on the $\mathrm{SH}$ coefficients of each model and the GRS80 normal gravity field parameters. Only the gravitational potential coefficients with harmonic degree $n \geq 2$ were considered for the SH-synthesis computations, thus excluding the contribution from the zero/first-degree harmonics. Note that EIGEN-CG01C and EIGEN-CG03C are the only models among the tested GGMs which are accompanied by non-zero first-degree SH coefficients (nevertheless their omission in the computation of the $N$ values has a negligible effect in our evaluation results).

The term $N_{o}$ represents the contribution of the zero-degree harmonic to the GGM-based geoid undulations with respect to the GRS80 reference ellipsoid. It is computed according to the well known formula (e.g. Heiskanen and Moritz 1967)

$N_{o}=\frac{G M-G M_{o}}{R \gamma}-\frac{W_{o}-U_{o}}{\gamma}$

where the parameters $G M_{o}$ and $U_{o}$ correspond to the Somigliana-Pizzeti normal gravity field generated by the GRS80 ellipsoid (Moritz 1992)

$$
\begin{aligned}
& G M_{o}=398600.5000 \times 10^{9} \mathrm{~m}^{3} \mathrm{~s}^{-2} \\
& U_{o}=62636860.85 \mathrm{~m}^{2} \mathrm{~s}^{-2}
\end{aligned}
$$

The Earth's geocentric gravitational constant (GM) and the constant gravity potential of the geoid $\left(W_{o}\right)$ have been set to the following values

$$
\begin{aligned}
& G M=398600.4415 \times 10^{9} \mathrm{~m}^{3} \mathrm{~s}^{-2} \\
& W_{o}=62636856.00 \mathrm{~m}^{2} \mathrm{~s}^{-2} \quad \text { (IERS Conventions 2003) }
\end{aligned}
$$

while the mean Earth radius $R$ and the mean normal gravity $\gamma$ on the reference ellipsoid are taken equal to $6371008.771 \mathrm{~m}$ and $9.798 \mathrm{~m} \mathrm{~s}^{-2}$, respectively (GRS80 values). Based on the above conventions, the zero-degree term from Eq. (3) yields the value $N_{o}=-0.442 \mathrm{~m}$, which has been added to the geoid undulations from all tested GGMs.

Note. The computation of the GGM geoid undulations from Eq. (2) was performed in the zero-tide system (with respect to a fixed reference ellipsoid GRS80).

\section{Height statistics}

The statistics of the individual height datasets that will be used in our evaluation tests are given in Table 2. Note that the statistics for the GGM geoid undulations refer to the values computed from Eq. (2) at the 1542 GPS/leveling benchmarks using the full spectral range of each model.

From Table 2, it is evident the existence of a large discrepancy $(>25 \mathrm{~cm})$ between the zero reference surface of the Hellenic vertical datum (which is associated with an unknown $W_{o}$ value) and the equipotential surface of the Earth's gravity field that is specified by $W_{o}=62636856.00 \mathrm{~m}^{2} \mathrm{~s}^{-2}$ and realized 
by the various geopotential models over the Greek mainland region.

Table 2. Statistics of the height datasets over the test network of $1542 \mathrm{GPS} /$ leveling benchmarks (units in meters).

\begin{tabular}{l|c|c|c|c}
\hline & Max & Min & Mean & $\sigma$ \\
\hline$h$ & 2562.753 & 24.950 & 545.676 & 442.418 \\
$H$ & 2518.889 & 0.088 & 510.084 & 442.077 \\
$N^{G P S}=h-H$ & 43.864 & 19.481 & 35.592 & 5.758 \\
\hline$N($ EGM08) & 44.374 & 19.663 & 35.968 & 5.800 \\
$N\left(\right.$ EGM08, $\left.n_{\max }=360\right)$ & 44.052 & 19.481 & 35.926 & 5.807 \\
$N($ EIGEN-GL04C) & 44.104 & 19.303 & 35.874 & 5.878 \\
$N($ EIGEN-CG03C) & 44.049 & 19.257 & 35.861 & 5.867 \\
$N($ EIGEN-CG01C) & 44.108 & 19.663 & 35.823 & 5.873 \\
$N($ GGM02C) & 44.034 & 19.771 & 35.905 & 5.780 \\
$N($ EGM96) & 44.007 & 19.687 & 36.037 & 5.753 \\
\hline \multicolumn{4}{|r}{}
\end{tabular}

It is interesting to observe the considerable mean offset of the full-resolution EGM08 geoid with respect to the geoid surface realized by the other geopotential models at the GPS/leveling benchmarks. This offset varies from 6 to $15 \mathrm{~cm}$ and it should be attributed to medium/long-wavelength systematic differences between EGM08 and the other GGMs over the Hellenic area.

\section{Pointwise tests}

The statistics of the differences between $N^{G P S}$ and the GGM geoid heights are shown in Table 3. In all cases, the values given in this table refer to the statistics after a simple (unweighted) least-squares constant bias fit was applied to the original misclosures $h-H-N$ at the 1542 GPS/leveling benchmarks. The significant variations in the estimated bias obtained from each tested model (see last column in Table 3) is an indication of large-scale systematic distortions among the GGM geoids, which are likely caused by medium/long-wavelength commission errors in their SH coefficients and additional omission errors in the pre-EGM08 models.

Table 3. Statistics of the differences $N^{G P S}-N$, after a leastsquares bias fit, at the $1542 \mathrm{GPS} /$ leveling benchmarks (units in meters).

\begin{tabular}{l|c|c|c|c}
\hline & Max & Min & $\sigma$ & Bias \\
\hline EGM08 $\left(n_{\max }=2190\right)$ & 0.542 & -0.437 & 0.142 & -0.377 \\
EGM08 $\left(n_{\max }=360\right)$ & 1.476 & -1.287 & 0.370 & -0.334 \\
EIGEN-GL04C $\left(n_{\max }=360\right)$ & 1.773 & -1.174 & 0.453 & -0.283 \\
EIGEN-CG03C $\left(n_{\max }=360\right)$ & 1.484 & -1.173 & 0.453 & -0.270 \\
EIGEN-CG01C $\left(n_{\max }=360\right)$ & 1.571 & -1.135 & 0.492 & -0.231 \\
GGM02C $\left(n_{\max }=200\right)$ & 2.112 & -1.472 & 0.551 & -0.313 \\
EGM96 $\left(n_{\max }=360\right)$ & 1.577 & -1.063 & 0.423 & -0.446 \\
\hline
\end{tabular}

The new EGM08 model offers a remarkable improvement in the agreement among ellipsoidal, orthometric and geoid heights over Greece. Compared to all other GGMs, the standard deviation of the EGM08 residuals $N^{G P S}-N$ decreases by a factor larger than 3. The improvement is evident even with the $30^{\prime}$ limited-resolution version $\left(n_{\max }=360\right)$ of the new model, which matches the GPS geoid within $\pm 37 \mathrm{~cm}$ in an average pointwise sense, while all previous GGMs of similar resolution do not perform better than $\pm 42 \mathrm{~cm}$. The major contribution, however, comes from the higher frequency band of EGM08 $(360<n<2190)$ which enhances the consistency between GGM and GPS geoid heights at $\pm 14 \mathrm{~cm}(1 \sigma$ level $)$.

The results of the pointwise evaluation have revealed that EGM08 performs exceedingly better than the other models over the mountainous parts of the test network. This is indicated from the scatter plots of the residuals $N^{G P S}-N$ (after the constant bias fit) with respect to the orthometric heights of the GPS/leveling benchmarks; see Fig. 2. These plots show a sizeable height-dependent bias between the GGM and the GPS geoid heights, which is considerably reduced in the case of EGM08. Apparently, the higher frequency content of the new model gives a better approximation for the terraindependent gravity field features over the Greek mainland, a fact that is clearly visible from the following plots.

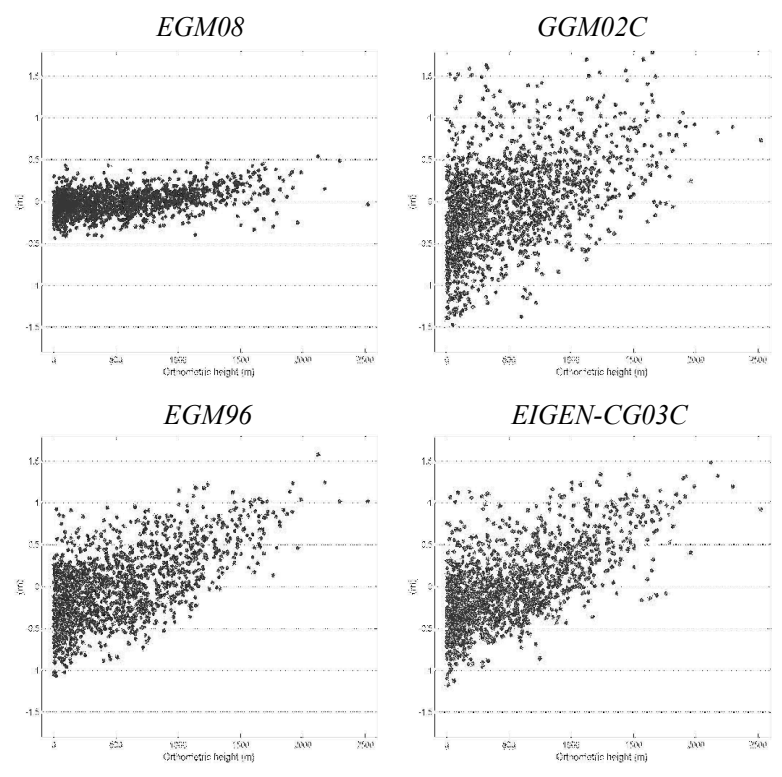

Figure 2. Height-dependent variations of the differences $N^{G P S}-N$ at the $1542 \mathrm{GPS} /$ leveling benchmarks.

Further manifestation of the strong correlation between the GGM/GPS geoid differences and the topographic height of the test points can be found in the color plots given in Kotsakis et al. (2008).

The spatial variations in the EGM08 residuals $N^{G P S}-N$ over the test network did not show any particular systematic pattern (apart from their overall 
dependency on the topography). Both the latitudedependent and longitude-dependent scatter plots of these residuals are free of any sizeable north/south or east/west tilts over the Greek mainland, as it can be seen in Fig. 3. In other GGMs, however, some strong localized tilts were identified in their undulation residuals with respect to the GPS geoid, mainly due to larger commission errors in their SH coefficients and significant aliasing errors resulting from their limited spatial resolution (see, e.g., the case of EGM96 shown in Fig. 3).
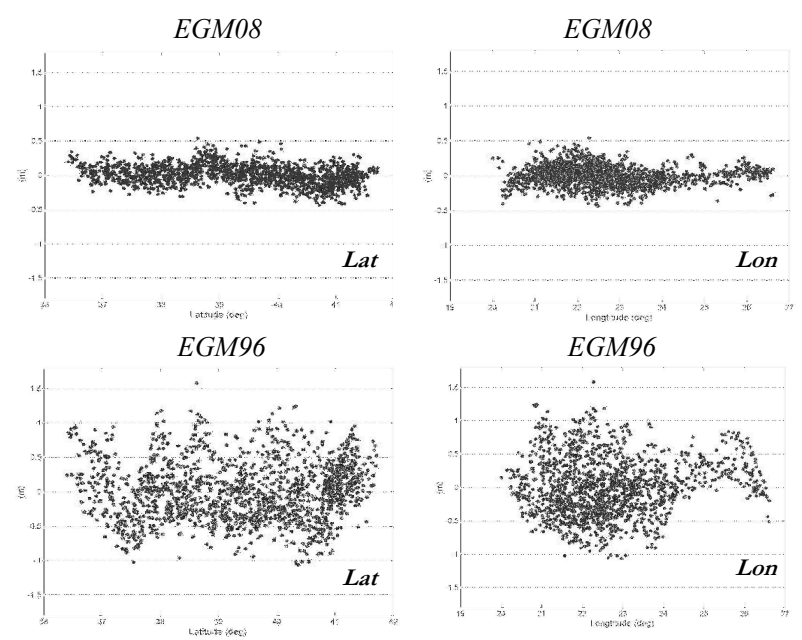

Figure 3. Latitude-dependent and longitude-dependent variations of the differences $N^{G P S}-N$ at the 1542 GPS/leveling benchmarks.

Overall, the EGM08 model outperforms all previous combined GGMs and it improves the statistical fit with the Hellenic GPS geoid by approximately $30 \mathrm{~cm}$ ! It is interesting to note that about $54 \%$ of the 1542 GPS/leveling benchmarks show an agreement between the EGM08 geoid and the GPS geoid that is better than $10 \mathrm{~cm}$, while almost $75 \%$ of the same points exhibit a consistency between the two surfaces that is better than $15 \mathrm{~cm}$. The corresponding percentages for the case of EGM96 decline to $18 \%$ and $28 \%$, respectively.

\section{Baseline tests}

In addition to the previous pointwise tests, another set of evaluation results was obtained through the comparison of GGM and GPS geoid-slope differences over the Hellenic network of 1542 GPS/leveling benchmarks. For all baselines formed within this network, the following relative undulation differences were determined

$$
\Delta N_{i j}^{G P S}-\Delta N_{i j}=\left(h_{j}-H_{j}-h_{i}+H_{i}\right)-\left(N_{j}-N_{i}\right)
$$

after the implementation of an initial least-squares bias/tilt fit between the pointwise GGM and the GPS geoid heights.

Depending on the actual baseline length, the computed values from Eq. (4) were grouped into various spherical-distance classes and their statistics were then evaluated within each class. Given the actual coverage and spatial density of the GPS/leveling benchmarks in our test network, baselines with length from $2 \mathrm{~km}$ up to $600 \mathrm{~km}$ were considered for this evaluation scheme.

The statistics of the differences between the GGM-based and the GPS-based geoid slopes are given in Tables 4 through 6 (for three selected baseline classes). As seen from these tables, the fullresolution EGM08 model performs consistently better than all other combined GGMs over all classes. The improvement becomes more pronounced for increasing baseline length, since the resultant $\sigma$ values are reduced by a factor of 1.4 for baselines $<5 \mathrm{~km}$, by a factor of 2 for baselines $5-10$ $\mathrm{km}$, and by a factor of 3.5 for baselines $10-50 \mathrm{~km}$.

Table 4. Statistics of the relative GGM/GPS geoid height differences for baselines with length $<5 \mathrm{~km}$ (number of baselines: 289 , units in meters).

\begin{tabular}{l|c|c|c|c}
\hline & Max & Min & $\sigma$ & Bias \\
\hline EGM08 $\left(n_{\max }=2190\right)$ & 0.643 & -0.474 & 0.111 & 0.006 \\
EGM08 $\left(n_{\max }=360\right)$ & 0.648 & -0.534 & 0.154 & 0.003 \\
\hline EIGEN-GL04C $\left(n_{\max }=360\right)$ & 0.649 & -0.542 & 0.155 & 0.005 \\
EIGEN-CG03C $\left(n_{\max }=360\right)$ & 0.643 & -0.540 & 0.155 & 0.005 \\
EIGEN-CG01C $\left(n_{\max }=360\right)$ & 0.640 & -0.536 & 0.156 & 0.005 \\
GGM02C $\left(n_{\max }=200\right)$ & 0.685 & -0.571 & 0.162 & 0.003 \\
EGM96 $\left(n_{\max }=360\right)$ & 0.643 & -0.553 & 0.154 & 0.005 \\
\hline
\end{tabular}

Table 5. Statistics of the relative GGM/GPS geoid height differences for baselines with length $5-10 \mathrm{~km}$ (number of baselines: 2119 , units in meters).

\begin{tabular}{l|c|c|c|c}
\hline & Max & Min & $\sigma$ & Bias \\
\hline EGM08 $\left(n_{\max }=2190\right)$ & 0.465 & -0.629 & 0.125 & 0.001 \\
EGM08 $\left(n_{\max }=360\right)$ & 1.022 & -1.044 & 0.248 & -0.004 \\
\hline EIGEN-GL04C $\left(n_{\max }=360\right)$ & 0.983 & -0.988 & 0.251 & -0.000 \\
EIGEN-CG03C $\left(n_{\max }=360\right)$ & 0.971 & -1.026 & 0.251 & -0.001 \\
EIGEN-CG01C $\left(n_{\max }=360\right)$ & 0.976 & -1.039 & 0.252 & -0.002 \\
GGM02C $\left(n_{\max }=200\right)$ & 0.967 & -0.991 & 0.264 & 0.002 \\
EGM96 $\left(n_{\max }=360\right)$ & 0.963 & -1.002 & 0.251 & 0.003 \\
\hline
\end{tabular}

Table 6. Statistics of the relative GGM/GPS geoid height differences for baselines with length $10-50 \mathrm{~km}$ (number of baselines: 56575, units in meters).

\begin{tabular}{l|c|c|c|c}
\hline & Max & Min & $\sigma$ & Bias \\
\hline EGM08 $\left(n_{\max }=2190\right)$ & 0.859 & -0.781 & 0.164 & -0.001 \\
EGM08 $\left(n_{\max }=360\right)$ & 2.778 & -2.417 & 0.514 & -0.012 \\
\hline EIGEN-GL04C $\left(n_{\max }=360\right)$ & 2.480 & -2.430 & 0.552 & -0.019 \\
EIGEN-CG03C $\left(n_{\max }=360\right)$ & 2.335 & -2.488 & 0.550 & -0.021 \\
EIGEN-CG01C $\left(n_{\max }=360\right)$ & 2.335 & -2.445 & 0.555 & -0.021 \\
GGM02C $\left(n_{\max }=200\right)$ & 3.221 & -2.760 & 0.627 & -0.012 \\
EGM96 $\left(n_{\max }=360\right)$ & 2.532 & -2.393 & 0.542 & -0.013
\end{tabular}


The overall behaviour of the $\sigma$ values for the GGM/GPS geoid slope differences is shown in Fig. 4 , over all baseline classes that were considered in our tests. The remarkable improvement in the relative undulation accuracy from the EGM08-based geoid is clearly visible, indicating an $\Delta N$ consistency level with the external GPS/leveling data that varies from $\pm 6 \mathrm{~cm}$ to $\pm 19 \mathrm{~cm}$ ( $1 \sigma$ level $)$.



Figure 4. Std of the differences $\Delta N_{i j}^{G P S}-\Delta N_{i j}$ in the network of 1542 GPS/leveling benchmarks, as a function of the baseline length.

Focusing on the $\Delta N$ evaluation results for short baselines (up to $30 \mathrm{~km}$ ) can give us an indication for the expected accuracy in GPS/leveling projects, when using an EGM08 reference geoid model over Greece. Our preliminary analysis in the test network showed that the agreement between the height differences $\Delta H_{i j}$ computed from: (a) the known orthometric heights and (b) the GPS/EGM08 ellipsoidal and geoid heights, can be approximated by the statistical error model $\sigma_{\Delta H}=\sigma_{o} L^{1 / 2}$ with $\sigma_{o}$ ranging between $3-5 \mathrm{~cm} / \mathrm{km}$ (for baseline length $L<30 \mathrm{~km}$ ). Although such a performance cannot satisfy $\mathrm{mm}$ level accuracy requirements for vertical positioning (which are 'easily' achievable through spirit leveling techniques), it nevertheless provides a major step forward that can successfully accommodate a variety of engineering and surveying applications. Note that the corresponding performance of EGM96 in our test network is described by a relative accuracy factor of $\sigma_{o} \approx 9 \mathrm{~cm} / \mathrm{km}$.

\section{Conclusions}

The results obtained from our evaluation study reveal the superiority of the new EGM08 model over all existing combined GGMs for the Hellenic area, and provide a promising testament for its future use in various geodetic applications in Greece.

Acknowledgements. The GPS and leveling data used for this study were kindly provided by Ktimatologio S.A under an ongoing research collaboration with the Department of Geodesy and Surveying (AUTh) in the frame of the HEPOS project.

\section{References}

Förste C, Flechtner F, Schmidt R, König R, Meyer U, Stubenvoll R, Rothacher M, Barthelmes F, Neumayer KH, Biancale R, Bruinsma S, Lemoine J-M (2006) A mean global gravity field model from the combination of satellite mission and altimetry/gravimetry surface gravity data. Poster presented at the EGU General Assembly, Vienna, Austria, April 2-7, 2006, Geophysical Research Abstracts, Vol. 8, 03462, 2006.

Förste C, Flechtner F, Schmidt R, Meyer U, Stubenvoll R, Barthelmes F, Rothacher M, Biancale R, Bruinsma S, Lemoine J-M (2005) A new high resolution global gravity field model from the combination of GRACE satellite mission and altimetry/gravimetry surface gravity data. Poster presented at the EGU General Assembly, Vienna, Austria, April 24-29, 2005, Geophysical Research Abstracts, vol. 7, 04561, 2005.

Gianniou M (2008) HEPOS: Designing and implementing an RTK network. Geoinformatics, 11(1): 10-13.

Heiskanen W, Moritz H (1967) Physical geodesy. WH Freeman, San Francisco.

Kotsakis C, Katsambalos K, Ampatzidis D, Gianniou M (2008) Evaluation of EGM08 in Greece using GPS and leveling heights. Presented at the IAG International Symposium on Gravity, Geoid and Earth Observation, Chania, Greece, June 23-27, 2008 (colored slides available at http://users.auth.gr/ kotsaki/Crete2008.pdf).

Lemoine FG, Kenyon SC, Factor JK, Trimmer RG, Pavlis NK, Chinn DS, Cox CM, Klosko SM, Luthcke SB, Torrence MH, Wang YM, Williamson RG, Pavlis EC, Rapp RH, Olson TR (1998) The Development of the Joint NASA GSFC and the National Imagery and Mapping Agency (NIMA) Geopotential Model EGM96. NASA Technical Paper NASA/TP1998206 861, Goddard Space Flight Center, Greenbelt, Maryland.

Moritz H (1992) Geodetic Reference System 1980. Bull Geod, 62(2): 187-192.

Pavlis NK, Holmes SA, Kenyon SC, Factor JK (2008) An Earth Gravitational Model to degree 2160: EGM2008. Presented at the 2008 General Assembly of the European Geosciences Union, Vienna, Austria, April 13-18, 2008.

Rapp RH (1997) Use of potential coefficient models for geoid undulation determinations using a spherical harmonic representation of the height anomaly/geoid undulation difference. J Geod, 71: 282-289.

Reigber C, Schwintzer P, Stubenvoll R, Schmidt R, Flechtner F, Meyer U, König R, Neumayer H, Förste C, Barthelmes F, Zhu SY, Balmino G, Biancale R, Lemoine J-M, Meixner H, Raimondo JC (2006) A high resolution global gravity field model combining CHAMP and GRACE satellite mission and surface data: EIGEN-CG01C. Scientific Technical Report STR06/07, GeoForschungsZentrum (GFZ), Potsdam.

Tapley BD, Ries J, Bettadpur S, Chambers D, Cheng M, Condi F, Gunter B, Kang Z, Nagel P, Pastor R, Pekker T, Poole S, Wang F (2005) GGM02 - An improved Earth gravity field from GRACE. J Geod, 79(8): 46747. 